\title{
The full penetration hole as a stochastic process: controlling penetration depth in keyhole laser-welding processes
}

\author{
A. Blug • F. Abt • L. Nicolosi - A. Heider • R. Weber • \\ D. Carl · H. Höfler $\cdot$ R. Tetzlaff
}

Received: 29 March 2012 / Revised version: 20 May 2012 / Published online: 5 July 2012

(C) The Author(s) 2012. This article is published with open access at Springerlink.com

\begin{abstract}
Although laser-welding processes are frequently used in industrial production the quality control of these processes is not satisfactory yet. Until recently, the "full penetration hole" was presumed as an image feature which appears when the keyhole opens at the bottom of the work piece. Therefore it was used as an indicator for full penetration only. We used a novel camera based on "cellular neural networks" which enables measurements at frame rates up to $14 \mathrm{kHz}$. The results show that the occurrence of the full
\end{abstract}

\author{
A. Blug $(\varangle) \cdot$ D. Carl $\cdot$ H. Höfler \\ Fraunhofer Institute for Physical Measurement Techniques IPM, \\ Heidenhofstr. 8, 79110 Freiburg, Germany \\ e-mail: Andreas.Blug@ipm.fraunhofer.de \\ Fax: +49-761-8857224 \\ D. Carl \\ e-mail: Daniel.Carl@ipm.fraunhofer.de \\ H. Höfler \\ e-mail: Heinrich.Hoefler@ipm.fraunhofer.de \\ F. Abt $\cdot$ A. Heider $\cdot$ R. Weber \\ IFSW Institut für Strahlwerkzeuge, Pfaffenwaldring 43, 70569 \\ Stuttgart, Germany \\ F. Abt \\ e-mail: Felix.Abt@ifsw.uni-stuttgart.de \\ A. Heider \\ e-mail: Andreas.Heider@ifsw.uni-stuttgart.de \\ R. Weber \\ e-mail: Rudolf.Weber@ifsw.uni-stuttgart.de \\ L. Nicolosi - R. Tetzlaff \\ IEE Institut für Grundlagen der Elektrotechnik u. Elektronik, \\ Mommsenstr. 12, 01069 Dresden, Germany \\ L. Nicolosi \\ e-mail: Leonardo.Nicolosi@tu-dresden.de \\ R. Tetzlaff \\ e-mail: Ronald.Tetzlaff@tu-dresden.de
}

penetration hole can be described as a stochastic process. The probability to observe it increases near the full penetration state. In overlap joints, a very similar image feature appears when the penetration depth reaches the gap between the sheets. This stochastic process is exploited by a closedloop system which controls penetration depth near the bottom of the work piece ("full penetration") or near the gap in overlap joints ("partial penetration"). It guides the welding process at the minimum laser power necessary for the required penetration depth. As a result, defects like spatters are reduced considerably and the penetration depth becomes independent of process drifts such as feeding rate or pollution on protection glasses.

\section{Introduction}

Laser beam welding is a joining technique frequently used in high volume applications, such as in the automotive industry. In so called keyhole welding processes, the laser beam is focused to intensities between $10^{6}$ and $10^{7} \mathrm{~W} / \mathrm{cm}^{2}$. In this regime, vaporization takes place on the surface of metals like steel or aluminum. This metal vapor forms the keyhole, i.e. a cavity within the melt pool, whose shape is defined by the balance between the vapor pressure and the surface tension of the surrounding melt [1]. Within the keyhole, the laser light is reflected multiple times resulting in a high absorption efficiency. As the laser beam advances, the melt flows around the keyhole and solidifies after a certain time. Therefore, deep and slender weld seams at high feeding rates are achieved. The disadvantage of this joining technique is the complexity of physical processes involved $[2,3]$. The efficiency of the process depends on a rather fragile equilibrium between the solid, liquid, and gaseous phase of the work 

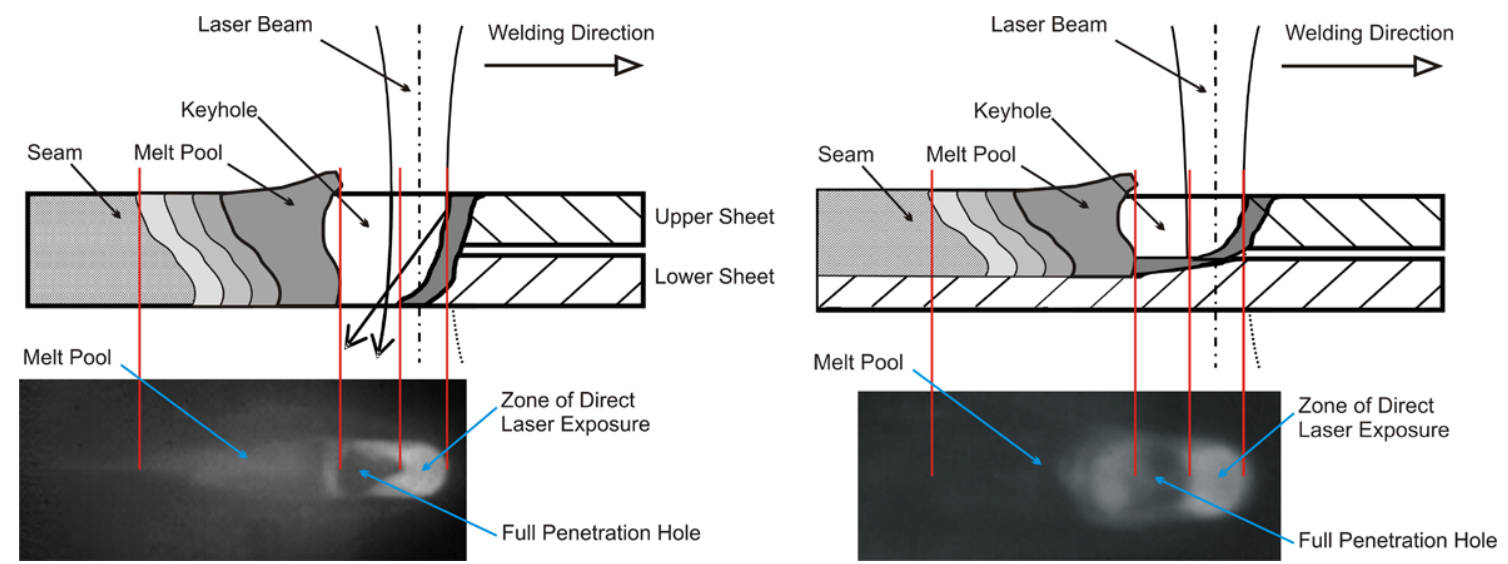

Fig. 1 Schematics of keyhole welding processes at "full penetration mode" (left) and "partial penetration mode" (right). The upper parts sketch cross sections of the welding processes, the lower parts show thermal images acquired coaxially to the laser axis

piece material. Therefore, perturbations such as the ejection of spatters can cause instabilities which result in weld seam defects such as craters, pores, seam under fill, bonding failure, or insufficient penetration depth [4].

In the left part of Fig. 1, such a keyhole penetration process in the full penetration mode is sketched for an overlap joint. As the laser beam moves from left to right, it forms a keyhole whose diameter is slightly bigger than the one of the laser beam. If a sufficient laser power is used, the depth of the keyhole reaches the bottom of the work piece and the so called full penetration hole (FPH) opens on the bottom side. At lower laser power values, the FPH closes again. The lower parts of Fig. 1 show the thermal images where the FPH appears as cool spots within the walls of the keyhole, which are at evaporation temperature of the work piece material. Thus, the appearance of the FPH indicates that the keyhole extends to the bottom of the lower sheet. The welding depth is defined as the depth of the liquid-solid interface, i.e. the depth of the molten material. Since the keyhole depth is usually slightly less than the welding depth, the FPH can be considered as an approximate measure for it.

The FPH at full penetration is a well-known image feature used for monitoring or closed-loop control of laser keyhole welding processes [5-7]. However, the FPH exhibits rapid fluctuations with frequencies of several $\mathrm{kHz}[8,9]$. Therefore, the approach of measuring the contrast of image intensity between the full penetration hole and the surrounding laser interaction zone at frame rates below $1 \mathrm{kHz}$ seemed inappropriate. For this reason, we introduced a novel camera technology based on so called "cellular neural networks" (CNN) in order to increase frame rate and robustness of the FPH detection. This component enables sampling rates which are sufficient to detect the stochastic properties of the process. The results of this novel approach for welds on zinc-coated steel sheets are presented in the following.
The second issue discussed in this paper is the partial penetration mode shown on the right hand side of Fig. 1 . In overlap joints, we observed an image feature very similar to the FPH because the vertical heat flow between the sheets is interrupted at the gap. Therefore, the surface of the lower sheet appears as a dark spot in the thermal image of the laser interaction zone. As discussed below, the statistics can be used to control penetration depth in the bottom sheet. Due to its similarity with the FPH in the full penetration state, we consider both image features as "full penetration holes" (FPH) in the following.

\section{System setup}

The key component of the system is a CNN-based camera. The CNN technology enables the integration of processor elements in the electronic circuitry of CMOS camera pixels. The result is a massively parallel computing architecture where a similar instruction is applied simultaneously to every camera pixel, a so called "Single Instruction, Multiple Data" (SIMD) system. Such systems are very efficient for real time image processing which is necessary for a robust FPH detection in highly dynamic processes [10]. Here, a system called Q-Eye was used consisting of $176 \times 144$ cells [11]. Each cell consists of a single photo sensor, processor and memory elements, and interconnections to the 8 neighboring cells. The Q-Eye is part of the Eye-RIS camera which contains an additional FPGA-based NIOS II processor by Altera which is used for the actuation tasks.

This CNN camera is mounted to the camera window of the welding head for a coaxial process observation (Fig. 2). For our experiments, the laser source was a $5 \mathrm{~kW}$, $1030 \mathrm{~nm}$ Trumpf TruDisk $5001 \mathrm{Yb}: Y A G$ thin disk laser with a $200 \mu \mathrm{m}$ transport fiber. The $2 \mathrm{D}$ laser scanner is integrated 
in the laser head (Trumpf PFO-33) which was equipped with a $450 \mathrm{~mm}$ focusing optics resulting in a focal diameter $l$ of $600 \mu \mathrm{m}$. The electrical signals are a $24 \mathrm{~V}$ digital start/stop signal and a $10 \mathrm{~V}$ analogue input to control the laser power with a bandwidth of $10 \mathrm{kHz}$. More details are provided in [12]. If not stated otherwise, the welding results shown below are obtained for overlap joints of zinc-coated steel sheets with a thickness of $1 \mathrm{~mm}$ and a gap width of $0.1 \mathrm{~mm}$.

For the acquisition of the thermal image the spectral range for the gray image is restricted to 820 to $980 \mathrm{~nm}$ where the thermal radiation is dominating. In this range, the keyhole walls appear very bright within the laser interaction zone because in first approximation they can be assumed as black body emitters at evaporation temperature of the work piece material. Therefore the intensity of the laser interaction zone is nearly independent of laser power and feeding rate.

For this configuration, a number of algorithms for the FPH detection were developed [13]. The constant intensity of the keyhole walls enables a contour detection of the FPH in the fluctuating melt pool. The frame rate is limited by the

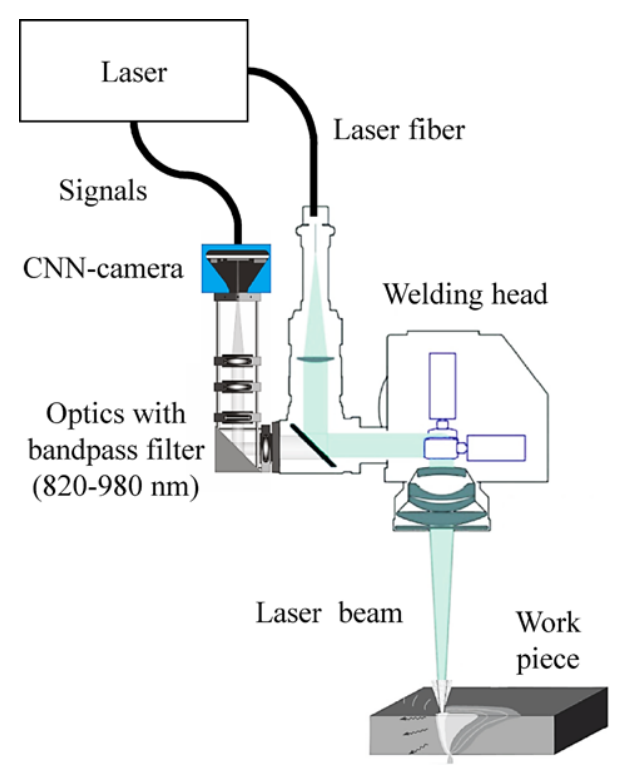

Fig. 2 System setup: The welding process is observed coaxially by the CNN camera mounted on the camera window of the welding head. The laser power is controlled over an analogue signal algorithm complexity which mainly depends on the assumptions made for the position of the FPH. The fastest detection was achieved for linear welds where the approximate position of the FPH is known. There, frame rates of up to $14 \mathrm{kHz}$ were achieved for image acquisition, image evaluation, and feedback. The latency time of the system is in the order of $100 \mu$ s. For welds with variable direction the frame rates decreased to $7 \mathrm{kHz}$. For every thermal image acquired at time $t_{i}$ the algorithms return a binary signal $s_{\mathrm{FPH}}\left(t_{i}\right)$ alternating between the values " 1 " if the FPH is detected or " 0 " otherwise.

\section{Characterization of the full penetration hole}

\subsection{Definition of measurement quantities}

Figure 3 shows the important elements from the control system point of view. These are the laser system, the welding process, the FPH detection by the CNN camera, and the feedback generation for the laser. Both, the laser system and the welding process are continuous in time and linked by the laser beam. The thermal image of the laser interaction zone is acquired by the CNN camera at discrete time steps $t_{i}=i / f$, where $i$ denotes the image number and $f$ the sampling rate of the camera. They are evaluated on the CNN for the presence of FPH events, resulting in the signal $s_{\mathrm{FPH}}\left(t_{i}\right)$. From this signal, including its history, a feedback $\Delta P\left(t_{i}\right)$ is generated in order to adapt the laser power to the current state of the process. In the open-loop system, the feedback is omitted. In this case, the laser power signal $P(t)$ forms the input and $s_{\mathrm{FPH}}\left(t_{i}\right)$ the output. In this way, the system can be regarded as a discrete time "Single Input, Single Output" (SISO) system.

In the following the signal $s_{\mathrm{FPH}}\left(t_{i}\right)$ is assumed to be a single outcome of a stochastic process in which the probability $p_{\mathrm{FPH}}\left(t_{i}\right)$ that a full penetration hole occurs at time $t_{i}$ depends on the energy previously coupled into the welding process by the laser power $P(t)$

$$
p_{\mathrm{FPH}}\left(t_{i}\right)=H(P(t), t) \quad \text { with } t \leq t_{i} .
$$

Due to the complexity of the welding processes, the operator $H$ is expected to be causal, nonlinear, and time dependentin particular in the presence of process perturbations. To
Fig. 3 Elements and signals of the closed-loop control system. For the open-loop characterization, $P(t)$ is the input signal and $s_{\mathrm{FPH}}(t)$ the output signal. The dashed line marks the components implemented in the $\mathrm{CNN}$ camera

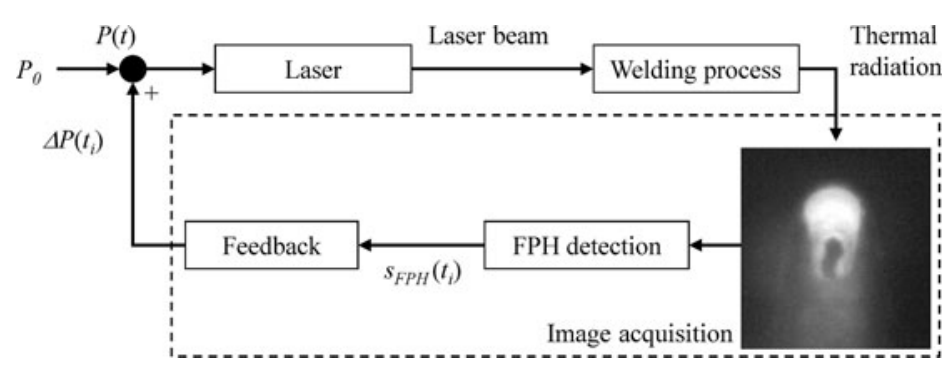


measure the probability $p_{\mathrm{FPH}}$ it is approximated as relative frequency either within an ensemble of signals at a fixed time $t$ or at different times $t_{i}$ within a single signal $s_{\mathrm{FPH}}\left(t_{i}\right)$. In the second case, the question of ergodicity must be considered [14]. Therefore we distinguish between relative frequencies $q_{\mathrm{FPH}}$ measured within an ensemble and relative frequencies $r_{\mathrm{FPH}}$ measured in time domain.

Considering the time scales, the reaction time of the welding process is the most difficult quantity to be estimated because of the complexity of the physics involved. For the step response, one obvious time constant is the value

$\tau_{l u} \equiv \frac{l}{u}$

where $l$ denotes the diameter of the laser beam and $u$ the feeding rate. This is the time during which the laser beam irradiates a single point on the weld seam. Thus, it is also the minimum time necessary for the welding process to reach stationarity after a stepwise change of the laser power $P(t)$. For a beam diameter $l$ of $0.6 \mathrm{~mm}$ and a feeding rate $u$ of $9 \mathrm{~m} / \mathrm{min}, \tau_{l u}$ is $4 \mathrm{~ms}$. The transport processes within the melt and the gas phase are much faster. In steel, the velocity of the convection within the melt can exceed the feeding rate $u$ by a factor of 10 and pressure waves within the gas phase travel at the speed of sound [15]. Therefore, the process can reach approximate values of the equilibrium point much faster. Since the reaction times of the laser system on the signal $P(t)$ and the delay of the CNN camera in the order of $100 \mu \mathrm{s}$ are negligible, the reaction time of the whole closed-loop system is dominated by the welding process and the value $\tau_{l u}$ might serve as a simplified measure for it.

The relation between the probability $p_{\mathrm{FPH}}\left(t_{i}\right)$ and the signal $s_{\mathrm{FPH}}\left(t_{i}\right)$ is modeled in the following way: The signal alternates between the discrete values 0 and 1 denoting the events "no FPH" and "FPH", respectively. Thus, the outcome $\xi=s_{\mathrm{FPH}}\left(t_{0}\right)$ at a distinct time $t_{0}$ within the process can be regarded as a random variable with the probabilities $p_{0} \equiv p_{\mathrm{FPH}}\left(t_{0}\right)$ for the events $\xi=1$ and $\left(1-p_{0}\right)$ for the event $\xi=0$. If the same process is repeated $n$ times, the $n$ outcomes $\xi_{1}, \ldots, \xi_{n}$ can be treated as $n$ independent Bernoulli trials where the probability to observe a number $n_{\mathrm{FPH}}$ of FPH events within the $n$ trials is given by the binominal distribution [14]. Thus, the probability $p_{0}$ can be estimated as the relative frequency $q_{\mathrm{FPH}}$ of FPH events at time $t_{0}$ within an ensemble of $n$ signals $s_{\mathrm{FPH}}\left(t_{0}\right)$

$q_{\mathrm{FPH}} \equiv \frac{n_{\mathrm{FPH}}}{n} \approx p_{0}$.

The number $n_{\mathrm{FPH}}$ is counted either by visual inspection of the thermal images at time $t_{0}$ or by an automatic evaluation using the $\mathrm{CNN}$ algorithm mentioned above. For the visual inspection, each image is classified as "FPH", "no FPH", or "uncertain" by experienced users. The criteria are the visibility of the FPH and the welding result obtained at time $t_{0}$.
Only the first two categories contribute to the number $n$ of images. In the case of an automatic evaluation, the CNN algorithm is used to count the number $n_{\mathrm{FPH}}$ of FPH events. The results of the visual inspection serve as a base for the optimization of the algorithm parameters. To obtain a value for the uncertainty of this estimation, the standard deviation $\sigma_{\mathrm{BIN}}$ of the Binominal distribution can be used

$\sigma_{\mathrm{BIN}}=\sqrt{n p_{0}\left(1-p_{0}\right)}$

The standard deviation $\sigma_{\mathrm{BIN}}$ is a measure for the width of the distribution of the $n_{\mathrm{FPH}}$ values around the expectation value. Applying error propagation to (3), the standard deviation of $q_{\mathrm{FPH}}$ becomes

$$
\begin{aligned}
\sigma_{\mathrm{FPH}} & =\frac{\partial q_{\mathrm{FPH}}}{\partial n_{\mathrm{FPH}}} \sigma_{\mathrm{BIN}}=\sqrt{\frac{p_{0}\left(1-p_{0}\right)}{n}} \\
& \approx \sqrt{\frac{q_{\mathrm{FPH}}\left(1-q_{\mathrm{FPH}}\right)}{n}}
\end{aligned}
$$

To illustrate the meaning and the limitations of this relation, one can derive a requirement for the sampling rate $f$ in ergodic processes from it. Since the reaction time of the closed-loop system is limited by the time $\tau_{l u}$ of the welding process, the uncertainty of the probability measurement depends on the number of images acquired with a sampling rate $f$ during this time. Therefore, a minimum frame rate $f_{\min }$ can be defined for a maximum standard deviation $\sigma_{\max }$

$f_{\min }=\frac{n}{\tau_{l u}}=\frac{p_{\mathrm{FPH}}\left(1-p_{\mathrm{FPH}}\right)}{\tau_{l u} \sigma_{\max }^{2}}$.

For a probability $p_{\text {FPH }}$ of $50 \%$, a reaction time $\tau_{l u}$ of $4 \mathrm{~ms}$, and a $\sigma_{\max }$ of $10 \%$ this equation requires a minimum frame rate $f_{\min }$ of $6.25 \mathrm{kHz}$. Of course, for Eqs. (4) to (6) independent Bernoulli trials are presumed. This independence is lost when the sampling rate of the camera is able to resolve the fluctuations in the melt pool. In this case, the true standard deviation is expected to be larger than the value $\sigma_{\mathrm{FPH}}$. Nevertheless, Eqs. (5) and (6) give some idea of the required sampling rates necessary for closed-loop control and of the uncertainty of the probability estimations during the openloop characterization.

\subsection{Open-loop characterization}

The aim of this section is to characterize the relation of Eq. (1) between the laser power $P(t)$ and the probability $p_{\text {FPH }}(t)$. This is done in the open loop, i.e. the feedback in Fig. 3 is omitted. $P(t)$ is used as input signal and $s_{\mathrm{FPH}}(t)$ as output signal. The probability $p_{\mathrm{FPH}}(t)$ is estimated by the relative frequency $q_{\mathrm{FPH}}$ defined in Eq. (3). Instead of repeating measurements $n$ times, periodic input signals $P(t)$ are used. In this case, images with the same phase $\omega t$ can be treated as independent if the duration $\tau_{P}=2 \pi \omega^{-1}$ of the period is sufficiently larger than the reaction time of the welding process estimated as $\tau_{l u}$. 
The most interesting relationship is the one between $p_{\mathrm{FPH}}$ and the laser power $P$ under stationary conditions. In order to decrease the number of measurements for a sufficient statistics, a slowly varying triangular input signal $P(t)$ is applied instead of a large number of measurements with constant laser powers. Afterwards, the images are classified into discrete laser power intervals of size $P_{I}$. The $p_{\text {FPH }}$ values measured with these triangular signals tend towards those measured under stationary conditions if the laser power variation within the time $\tau_{l u}$ is smaller than the interval size $P_{I}$. For example, if $P_{I}$ is $100 \mathrm{~W}$, the slope $\dot{P}(t)$ of the triangular signal $P(t)$ is chosen so that the product $|\dot{P}| \tau_{l u}$ is smaller. Images acquired within the same power interval are combined to a class. The number of thermal images with FPH is counted by visual inspection.

Figure 4 shows results measured at four different feeding rates between 3 and $9 \mathrm{~m} / \mathrm{min}$ on an overlap joint of two $1 \mathrm{~mm}$ zinc-coated steel sheets. For a laser power interval $P_{I}$ of $100 \mathrm{~W}$ about 60 images were evaluated per class and feeding rate. In order to make the results for the different feeding rates comparable, they are drawn over the line energy $P / u$ which is the laser energy irradiated per welding length. At line energies above $50 \mathrm{~J} / \mathrm{mm}$, full penetration is achieved. At line energies between 50 and $70 \mathrm{~J} / \mathrm{mm}, q_{\mathrm{FPH}}$ increases almost linearly from $18 \%$ to $75 \%$, as marked by the straight line. At higher line energies, a saturation value of about $80 \%$ was found. Since defects like spatters or smoke residues are caused by an excess energy, the linear range marked by the straight line is preferred for full penetration. If the welding process is ergodic, the rate of FPH events can be used as a set point for the closed-loop control.

Whereas the full penetration behavior is similar for butt joints and overlap joints, the peak around $30 \mathrm{~J} / \mathrm{mm}$ only occurs at overlap joints. At this line energy, the bottom of the vapor capillary reaches the interface between the top and the

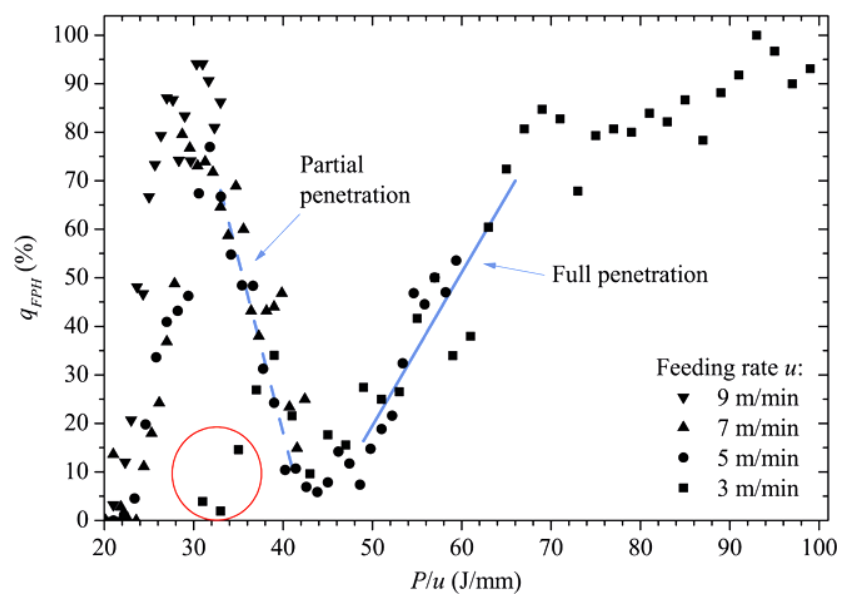

Fig. 4 Relationship between the relative frequency $q_{\mathrm{FPH}}$ of FPH events and the line energy $P / u$. The straight line marks the shoulder at full penetration, the dashed one at partial penetration bottom sheets. Since the vertical heat flux between the two sheets is interrupted at the gap, the upper surface of the bottom sheet, which is not directly irradiated by the laser beam, remains colder than the surrounding keyhole walls which lie within the upper sheet. Therefore, the bottom of the keyhole appears as a dark spot within the thermal image. In the rising shoulder between 20 and $30 \mathrm{~J} / \mathrm{mm}$ the keyhole penetrates the upper sheet and reaches the gap only occasionally. In the falling shoulder (dashed line) the probability for FPH events decreases because the keyhole penetrates the bottom sheet and lowers the temperature difference between the two sheets.

In Fig. 4, the three data points in the curve acquired at $u=3 \mathrm{~m} / \mathrm{min}$ and $\mathrm{P} / \mathrm{u}<36 \mathrm{~J} / \mathrm{mm}$ are marked by a circle because they differ significantly from those curves acquired at $u=5$ and $7 \mathrm{~m} / \mathrm{min}$. To find the cause, the relative frequencies measured at the rising and falling slopes of the triangular laser power signal were separated. Figure 5 shows data of the curves acquired at $u=3$ and $5 \mathrm{~m} / \mathrm{min}$. At $u=3 \mathrm{~m} / \mathrm{min}$, FPH events occur at the gap only at rising laser power $P$. At higher feeding rates, similar $q_{\mathrm{FPH}}$ values were observed in both branches at line energies between 30 and $40 \mathrm{~J} / \mathrm{mm}$. At line energies below $30 \mathrm{~J} / \mathrm{mm}$, the majority of FPH events occur in the falling branch. A possible explanation for the behavior of the curve at $u=3 \mathrm{~m} / \mathrm{min}$ is that the velocity of the horizontal heat flux is comparable to or higher than the feeding rate. Therefore, the bottom sheet remains hot at falling laser powers and no FPH is visible. At higher feeding rates, the influence of the horizontal heat flux is negligible. The hysteresis effect at line energies below $30 \mathrm{~J} / \mathrm{mm}$ is more difficult to understand. Apparently, the keyhole opens at line energies of about $30 \mathrm{~J} / \mathrm{mm}$, but once it is open, it remains in that state until line energy drops below $25 \mathrm{~J} / \mathrm{mm}$. A physical cause of this behavior might be the surface tension of the melt [16].

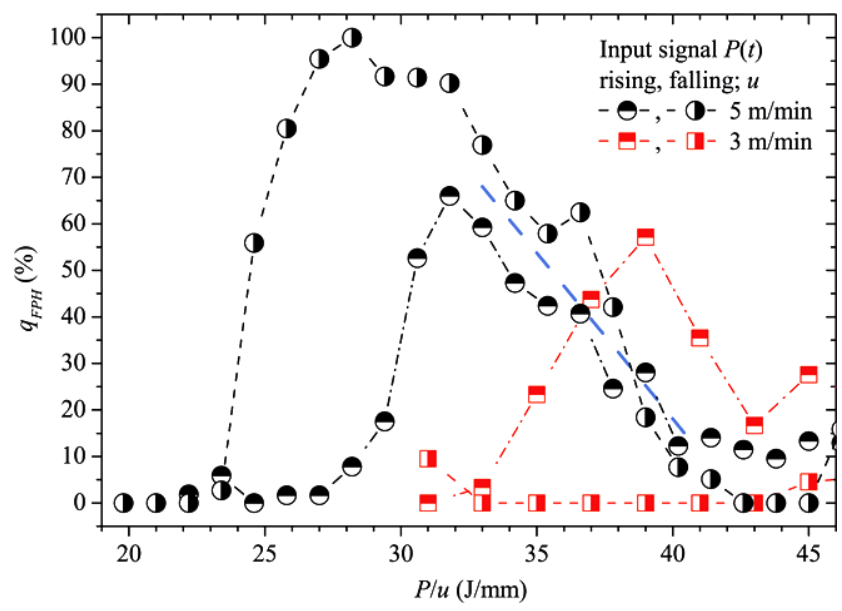

Fig. 5 Comparison of the relative frequencies $q_{\mathrm{FPH}}$ measured at rising and falling laser powers. The dashed line marks the partial penetration regime from Fig. 4 

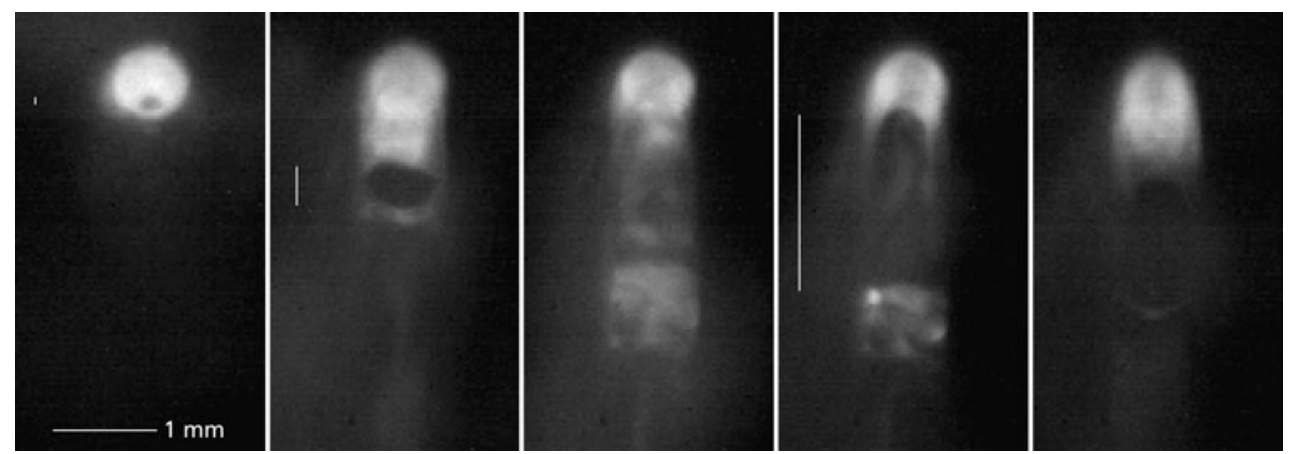

Fig. 6 Development of the thermal images during the acquisition of Fig. 4 at $u=5 \mathrm{~m} / \mathrm{min}$. The vertical lines mark the FPH. From left to right: FPH at partial penetration with rising laser power at $2.5 \mathrm{~kW}$ $(30 \mathrm{~J} / \mathrm{mm}), \mathrm{FPH}$ at full penetration near $5 \mathrm{~kW}(60 \mathrm{~J} / \mathrm{mm})$, melt pool at

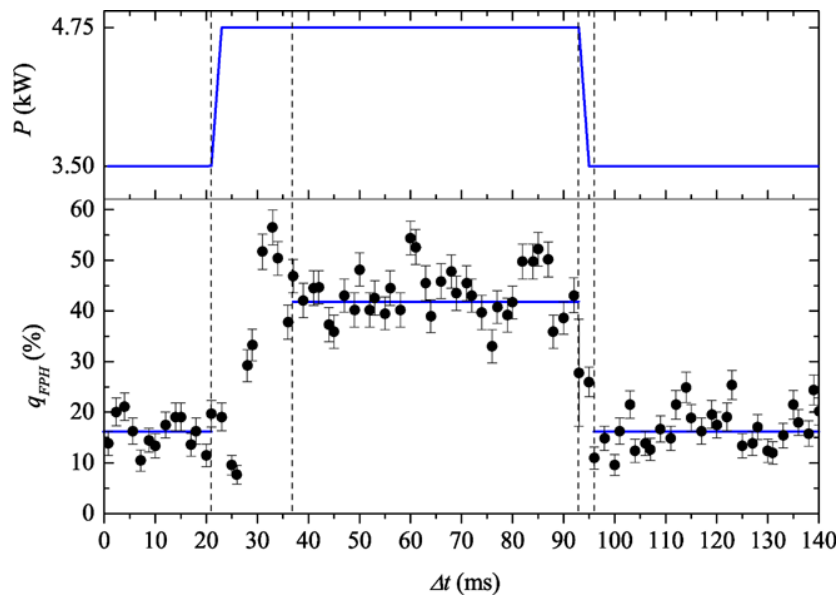

Fig. 7 Response of $q_{\mathrm{FPH}}$ to a rectangular input signal $P(t)$. The dashed lines mark the transient regimes. The response time of the welding process is about $10 \mathrm{~ms}$ at the rising and less than $5 \mathrm{~ms}$ at the falling edge

This behavior is also visible in the thermal images of the melt shown in Fig. 6. At the rising branch, the keyhole is very short with a small FPH. At full penetration, keyhole length and FPH size increase. At falling laser powers, keyhole length and FPH increase dramatically. With the size of the keyhole, the temporal response of the welding process can be expected to change, too. Therefore, these measurements indicate that the dynamic properties of the welding process depend not only on the absolute value of the laser power but also on the way how the process is guided.

The second important issue is the characterization of the transient behavior of the probability $p_{\mathrm{FPH}}(t)$. For this purpose, a periodic rectangular laser power signal $P(t)$ with period $\tau_{P}$ is applied to the open-loop system. From the different periods, images with similar phase $\varphi=\omega t$ with $\omega=2 \pi \tau_{P}^{-1}$ are combined to a class. In order to obtain a sufficient statistics, the automatic evaluation result of the linear $\mathrm{CNN}$ algorithm was used. Figure 7 shows the result of the falling laser power near $3.3 \mathrm{~kW}(40 \mathrm{~J} / \mathrm{mm})$, FPH at partial penetration near $3.1 \mathrm{~kW}(37 \mathrm{~J} / \mathrm{mm})$ with a widely opened keyhole, and closed FPH at $2.0 \mathrm{~kW}(24 \mathrm{~J} / \mathrm{mm})$

automatic evaluation of 19,039 images acquired with a rectangular signal $P(t)$ with period $\tau_{P}$ of $140 \mathrm{~ms}$. This period is divided into 89 classes where the phase shift $\Delta \varphi$ within each class corresponds to a time shift $\Delta t=\Delta \varphi \tau_{P} / 2 \pi \approx 1.5 \mathrm{~ms}$. The resulting average class size is 214 images. The probability to observe a FPH within each class is again estimated by calculating the $q_{\mathrm{FPH}}$ values according to Eq. (3).

Figure 7 shows the $q_{\mathrm{FPH}}$ values calculated for each class together with one period of the input signal $P(t)$. The laser power alternates between 3.5 and $4.75 \mathrm{~kW}$. The dots mark the $q_{\mathrm{FPH}}$ values calculated for each class. The length of the error bars is the standard deviation $\sigma_{\mathrm{FPH}}$ calculated according to Eq. (5). Starting at the rising edge of the laser power signal, the graph can be represented by four regions: Between $\Delta t=21$ and $36 \mathrm{~ms}$, transient behavior of $q_{\mathrm{FPH}}$ is observed. Between $\Delta t=36 \mathrm{~ms}$ and the falling edge at $\Delta t=93 \mathrm{~ms}$, the $q_{\mathrm{FPH}}$ values scatter around a constant value of about $42 \%$. At the falling edge, a second transient region is observed. In contrast to the transient region at the rising edge, this region is significantly shorter. After about $3 \mathrm{~ms}$, another region with approximately constant $q_{\mathrm{FPH}}$ values was observed in the range between $\Delta t=96 \mathrm{~ms}$ and $140 \mathrm{~ms}$ of one period and $\Delta t=0$ to $21 \mathrm{~ms}$ of the next period. Due to the reduced laser power of $3.5 \mathrm{~kW}$, full penetration holes are measured with an average probability of about $16 \%$. A very similar behavior was observed for aluminum welds [12].

We interpret these measurements in the following way: After a transient response time, the $q_{\mathrm{FPH}}$ values become constant which means that the process is "wide sense stationary" [14]. On a time scale larger than this "relaxation time", the process can be expected to become ergodic if no perturbations are present [17]. At the rising edge, this "relaxation time" is about $15 \mathrm{~ms}$ and therefore longer than $\tau_{l u}$ which is $7.5 \mathrm{~ms}$ for a feeding rate of $5 \mathrm{~m} / \mathrm{min}$. On the falling edge, the transient regime vanishes already after $3 \mathrm{~ms}$, which can be explained by the transportation speed in the liquid and gaseous phase (see Sect. 3.1). The difference in the length 
of the transient regimes at the rising and the falling edge clearly shows that the operator $H$ in Eq. (1) must be nonlinear or time dependent. To proof this formally, one can define a new input signal

$$
P_{S}(t) \equiv P_{R}(t)+P_{F}(t)=\text { const }
$$

with $P_{R}(t) \equiv P\left(t-t_{R}\right)$ and $P_{F}(t) \equiv P\left(t-t_{F}\right)$, where $t_{R}=$ $21 \mathrm{~ms}$ and $t_{F}=93 \mathrm{~ms}$ are the start times of the rising and falling edges in the laser power signal of Fig. 7. Assuming the operator $H$ to be linear and time invariant, one must also expect the sum of the output signals $p_{R}(t) \equiv H\left(P_{R}(t)\right)$ and $p_{F}(t) \equiv H\left(P_{F}(t)\right)$ to be constant, because

$$
\begin{aligned}
p_{S}(t) & \equiv p_{R}(t)+p_{F}(t)=H\left(P_{R}(t)\right)+H\left(P_{F}(t)\right) \\
& =H\left(P_{R}(t)+P_{F}(t)\right)=\text { const } .
\end{aligned}
$$

Figure 8 shows the result of this test for the data from Fig. 7 . Whereas the sum $P_{S}(t)$ of the shifted laser power signals is constant, the sum of $q_{\mathrm{FPH}}\left(t-t_{R}\right)$ and $q_{\mathrm{FPH}}\left(t-t_{F}\right)$ shows a minimum at $\Delta t=6 \mathrm{~ms}$. In the constant regimes the sum of the $q_{\mathrm{FPH}}$ signals is about $60 \%$ (dashed line). At the minimum, this sum drops to $21 \%$ with a standard deviation $\sigma_{S} \equiv \sqrt{\sigma_{\mathrm{FPH}}^{2}\left(t-t_{R}\right)+\sigma_{\mathrm{FPH}}^{2}\left(t-t_{F}\right)}$ of about $4 \%$. Even if $\sigma_{\mathrm{FPH}}$ underestimates the true uncertainty due to the high frame rate, this drop from 60 to $21 \%$ is significant. Therefore $H$ cannot be linear and time invariant which means that most of the standard feedback strategies are not applicable. Considering the complexity of the welding process, this finding is not surprising. Nonlinear dependencies were also found for the relation between penetration depth and melt pool intensity [18] and between penetration depth and melt pool width [19].

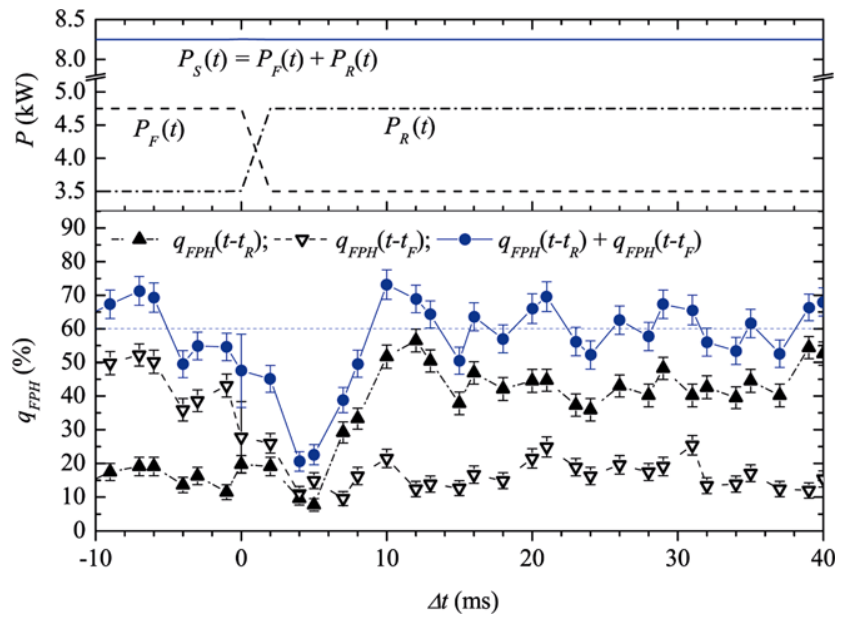

Fig. 8 Proof of nonlinearity with data from Fig. 7. A constant input signal $P_{S}(t)$ results in a time dependent output signal $q_{\mathrm{FPH}}\left(t-t_{R}\right)+q_{\mathrm{FPH}}\left(t-t_{F}\right)$

\subsection{Closed-loop control}

For the closed-loop system sketched in Fig. 3, a feedback strategy is required which is robust against the transient response of the process. In contrast to publications [18, 19] which apply a time independent nonlinear model to images acquired at rather low frame rates, we exploit the high frame rate and short latency achieved by the CNN camera. Of course, the probability $p_{\mathrm{FPH}}(t)$ has to be estimated within a single sample function which means that the relative frequency must be measured at different times. This section discusses the equilibrium states achieved by this control strategy for ergodic processes without perturbations. Under this assumption one can estimate $p_{\mathrm{FPH}}(t)$ by the number $n_{\mathrm{FPH}}\left(t, \tau_{S}\right)$ of $\mathrm{FPH}$ events observed within $n\left(t, \tau_{S}\right)$ images sampled within a time interval $\left[t-\tau_{S}, t\left[\right.\right.$ with $\tau_{S}=n / f$. The result is a rate $r_{\mathrm{FPH}}(t)$ of $\mathrm{FPH}$ events with

$r_{\mathrm{FPH}}\left(t, \tau_{S}\right) \equiv \frac{n_{\mathrm{FPH}}\left(t, \tau_{S}\right)}{n\left(t, \tau_{S}\right)} \approx p_{\mathrm{FPH}}(t)$.

A simple strategy is to start with an initial value $P_{0}$ and to adapt this value after every acquired image. If no FPH is observed, a value $\Delta P_{\text {up }}$ is added to the current laser power $P(t)$; otherwise, a value $\Delta P_{\text {down }}$ is subtracted. As shown in Fig. 4, the probability to detect a FPH rises with increasing laser power $P$ in the regime of full penetration. Therefore, such a system reaches its operating point, i.e. the average laser power over a time interval $\tau_{S} \gg \tau_{l u}$ is constant when the following equilibrium condition is fulfilled:

$r_{\mathrm{FPH}} \Delta P_{\text {down }}=\left(1-r_{\mathrm{FPH}}\right) \Delta P_{\text {up }}$.

The time variables $t$ and $\tau_{S}$ are omitted because $r_{\mathrm{FPH}}$ becomes time independent in the state of equilibrium. The left side of this equation describes the frequency with which the laser power is lowered, the right side the frequency of raising the laser power. The sign of both, $\Delta P_{\text {up }}$ and $\Delta P_{\text {down }}$ is chosen positive. Resolving Eq. (10) for $r_{\mathrm{FPH}}$, one obtains the set point

$r_{F P} \equiv \frac{\Delta P_{\text {up }}}{\Delta P_{\text {up }}+\Delta P_{\text {down }}}$.

In the regime of partial penetration marked by the dashed line in Fig. 4, the probability to detect a FPH decreases with increasing laser power $P$. This means that the laser power must be increased by $\Delta P_{\text {up }}$ in case of an FPH and decreased by $\Delta P_{\text {down }}$ if no FPH is detected. Therefore, the laser power rises with the probability $r_{\mathrm{FPH}}$ and drops with the probability $\left(1-r_{\mathrm{FPH}}\right)$. So in partial penetration, the equilibrium is reached at the set point

$r_{P P} \equiv \frac{\Delta P_{\text {down }}}{\Delta P_{\text {up }}+\Delta P_{\text {down }}}$.

This control strategy together with measurement results was described in [9]. It was shown that such a system oscillates 
around an average laser power $P_{S}$. However, we found that the amplitude of the oscillations depends very much on the feeding rate. The control behavior of this simple strategy was not satisfactory, because it was either slow or resulted in large oscillation amplitudes. The reason for this behavior is the excess energy brought into the process during its reaction time. This excess energy was reduced by limiting the oscillation amplitudes with boundaries. These boundaries remain constant as long as the process oscillates around the set point. As soon as the system stops oscillating, the boundaries are adapted in appropriate steps. In this way, the reaction speed of the closed-loop system to large perturbations is decoupled from the oscillation amplitude under constant process conditions. Details are described in [20]. Figure 9 shows the FPH signal $s_{\mathrm{FPH}}(t)$ and the corresponding laser power signal $P(t)$ for a welding process under constant process conditions with feeding rate $5 \mathrm{~m} / \mathrm{min}$. The laser power signal is clipped at the boundaries (dashed lines) if the amplitude of the oscillations exceeds $\pm 100 \mathrm{~W}$. This clipping limits the excess energy brought into the process during its reaction time which makes the amplitudes independent of the feeding rate. Under constant process conditions, the

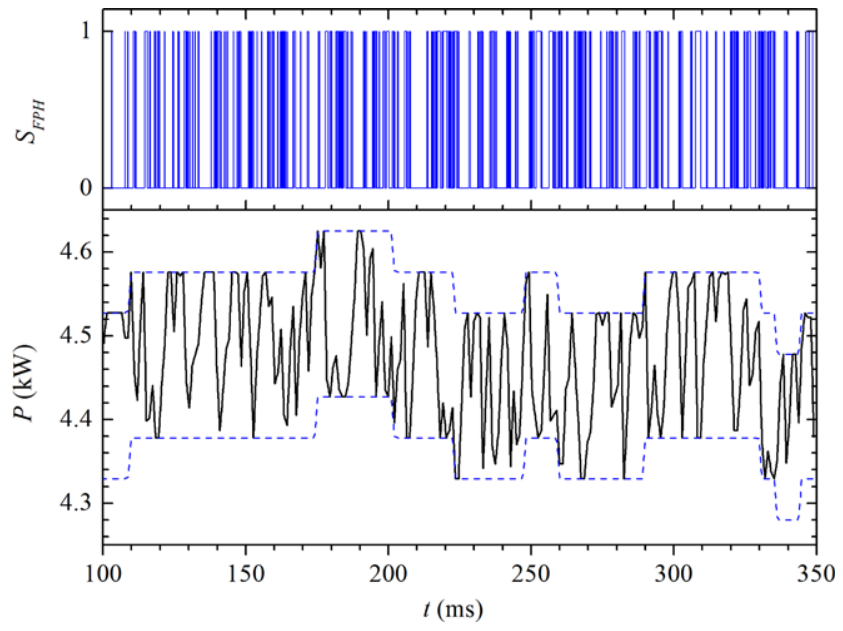

Fig. 9 Signal $s_{\mathrm{FPH}}(t)$ for the detection of FPH events and feedback $P(t)$ in a closed-loop controlled welding process under constant process conditions. The limits for the laser power (dashed lines) limit the excess energy within the process. They are adapted due to the oscillations

Fig. 10 Bottom weld seams of controlled full penetration welds (feeding rate $5 \mathrm{~m} / \mathrm{min}$, length $80 \mathrm{~mm}$, focal width $0.6 \mathrm{~mm}$ ) with set point $r_{F P}=50 \%$ (top, $3.8 \mathrm{~kW})$ and $r_{F P}=25 \%$ (bottom, $3.6 \mathrm{~kW}$ ) boundary varies only by a single step. In the case of a perturbation which alters penetration depth, oscillation stops and laser power is adapted in the rising or falling direction until oscillations start again. In practical applications, the laser power is adapted with a speed of about $30 \mathrm{~kW} / \mathrm{s}$ [21].

Although both signals in Fig. 9 are acquired within the same loop, the autocovariance $C(\tau)$ of the $s_{\mathrm{FPH}}$ signal decreases significantly faster than the one of the laser power signal. The correlation time $\tau_{C}$ defined as

$\tau_{C} \equiv \frac{1}{C(0)} \int_{0}^{\infty} C(\tau) d \tau$

is estimated to be $3 \mathrm{~ms}$ for the signal $s_{\mathrm{FPH}}(t)$ and $8 \mathrm{~ms}$ for $P(t)$. The reason for this difference can be seen in Fig. 9 where the signal $s_{\mathrm{FPH}}(t)$ fluctuates much quicker than $P(t)$ which supports the assumption that the probability $p_{\mathrm{FPH}}$ is a stochastic quantity for the state of the process. Please note that the correlation time of the laser signal is very close to the time constant $\tau_{l u}$ of $7.2 \mathrm{~ms}$.

As this measurement shows, the rate $r_{\mathrm{FPH}}$ of FPH events can be used to control the average laser power as presumed in Eqs. (10) to (12). In the "full penetration" regime the average laser power increases with increasing set point $r_{F P}$. In this regime, the degree of full penetration can be selected

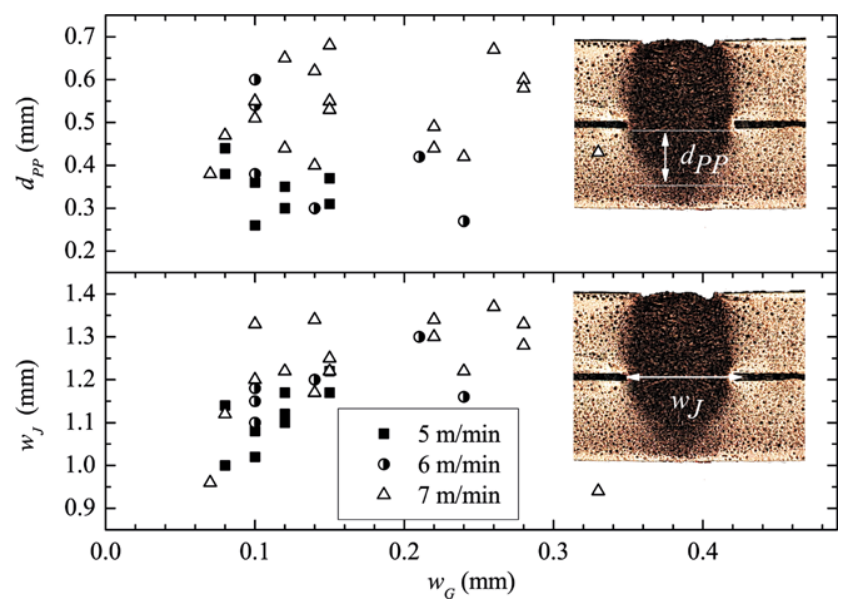

Fig. 11 Dependence of penetration depth $d_{P P}$, and joint width $w_{J}$ of partial penetration welds on the gap width $w_{G}$ at set point $r_{P P}=33 \%$ and at different feeding rates $u$
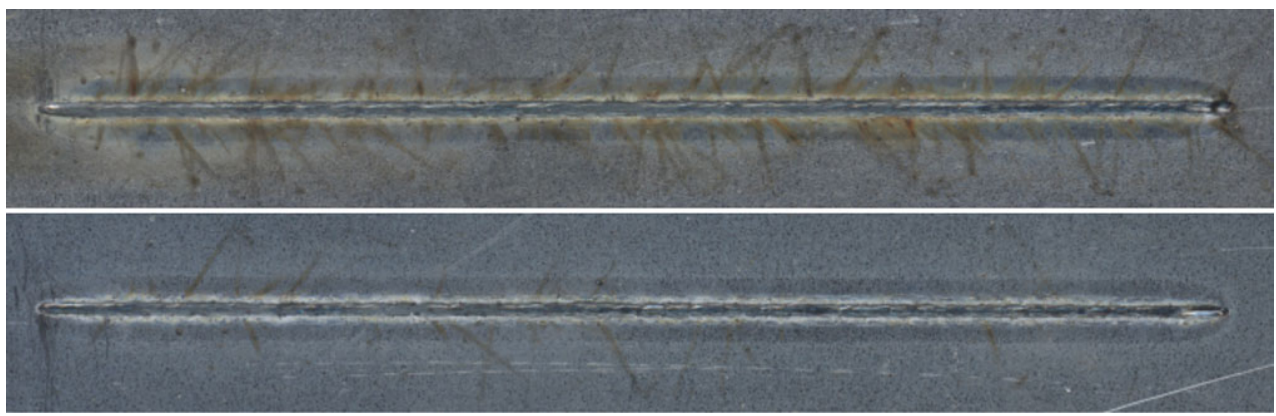
with this set point as shown in Fig. 10. At $r_{F P}=50 \%$, the average laser power is $3.8 \mathrm{~kW}$. So the closed-loop system guides the process at the minimum laser power necessary for certain degree of penetration which is defined by the set point $r_{F P}$. This reduces the energy in the process and therefore spatters and smoke residues at the bottom weld seam.

In the "partial penetration" regime, the set point $r_{P P}$ also defines the average laser power which increases in this regime with decreasing values of $r_{P P}$ (dashed line in Fig. 4). For a sheet thickness of $1 \mathrm{~mm}$, we achieved good partial penetration welds at set points $r_{P P}$ between $25 \%$ corresponding to an average power of $3.7 \mathrm{~kW}$ and $50 \%$ corresponding to $3.3 \mathrm{~kW}$. Within this range, a set point for desired penetration depth can be chosen. Figure 11 shows the dependence of the joint geometry parameters penetration depth $d_{P P}$ and joint width $w_{J}$ on the gap width $w_{G}$ and on the feeding rate $u$. These parameters are measured at 34 cross sections of partial penetration welds acquired with the same set point $r_{P P}$ of $33 \%$. One can see that the penetration depth $d_{P P}$ does not vary significantly with the gap width $w_{G}$, but there is a slight dependence on the feeding rate $u$ : it rises from $0.35 \mathrm{~mm}$ at $u=5 \mathrm{~m} / \mathrm{min}$ to $0.52 \mathrm{~mm}$ at $u=7 \mathrm{~m} / \mathrm{min}$. The second important parameter for the determination of the seam strength is the joint width $w_{J}$ within the gap. For a gap size between 0.1 and $0.3 \mathrm{~mm}$, it is about $1.2 \mathrm{~mm}$. Outside of this range, the joint width decreases to roughly $1 \mathrm{~mm}$. Therefore, the value of $r_{P P}$ can be considered as an indirect

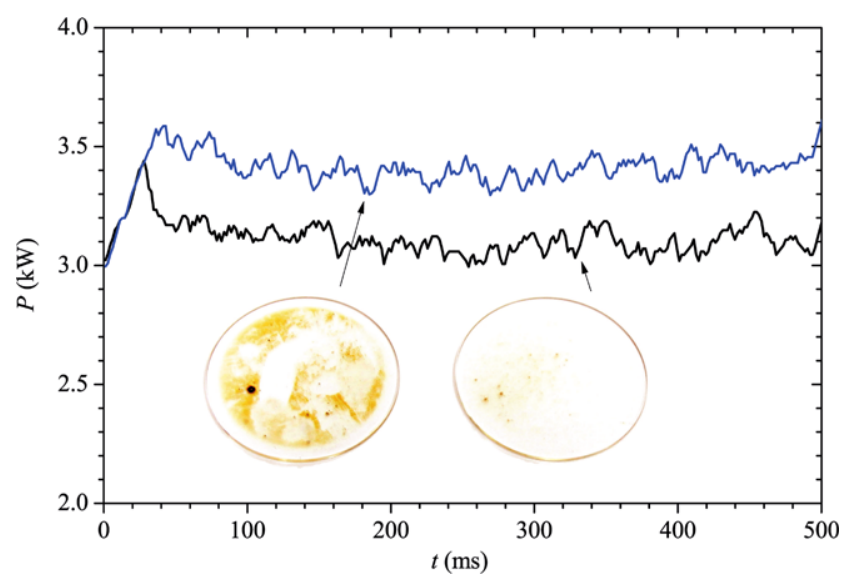

Fig. 12 Laser power signals $P(t)$ for penetration welds with a clean and a polluted protection glass with focal diameter $l=0.2 \mathrm{~mm}$ and feeding rate $u=9 \mathrm{~m} / \mathrm{min}$ set point for the penetration depth $d_{P P}$ at a given feeding rate. More details are discussed in [22].

\section{Welding results}

The previous results show the properties of the closed-loop control under constant conditions. For practical applications, one must proof that the system is able to compensate process drifts. To test these effects, a large number of experiments were carried out. They show that in addition to the reduction of spatters and smoke residues the system is able to compensate process drifts like variations in sheet thickness, focal drifts, or cross jet variations in a large range. Figures 12 and 13 show the reaction of the closed-loop system to a typical drift occurring during production: laser light is scattered on polluted protection glasses. The only difference between the two welds is that one was carried out with a clean protection glass, the other one with a polluted protection glass. The closed-loop system automatically raises the laser power so that virtually no difference is visible between the resulting bottom weld seams in Fig. 13.

The closed-loop control system also compensates variations in the feeding rate as they occur for example in robot welding. Figure 14 shows a partial penetration weld where the laser power was automatically adapted. As it can be ex-

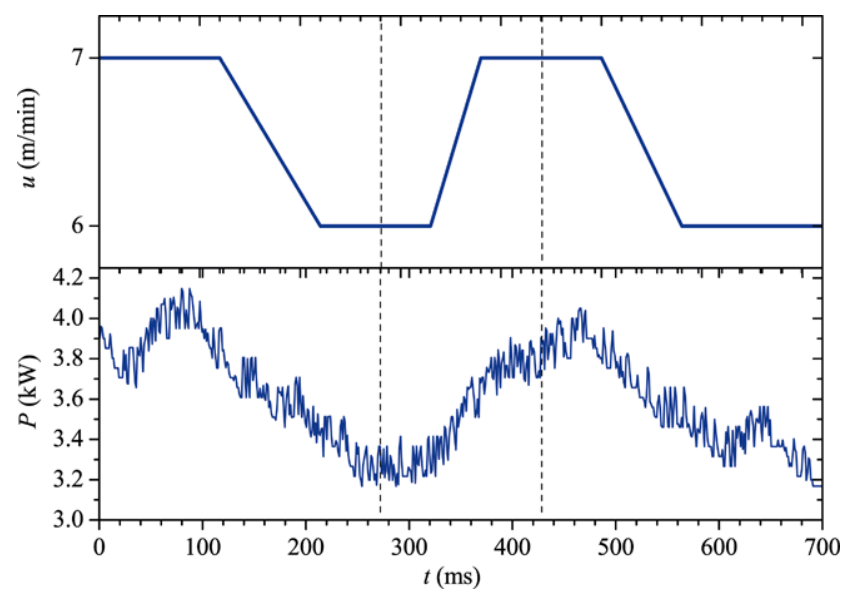

Fig. 14 Partial penetration weld with variable feeding rate $u$. Laser power $P$ is adapted automatically. The dashed lines mark the approximate position of the upper cross sections in Fig. 15
Fig. 13 Bottom weld seams to Fig. 12 with clean (top) and polluted (bottom) protection glass. The effect of the pollution is compensated by the closed-loop control

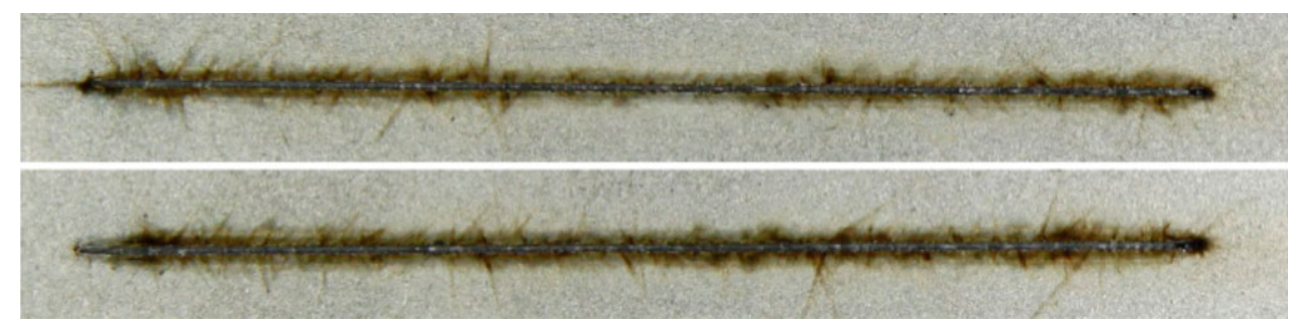


Fig. 15 Cross sections at the dashed lines in Fig. 14 at $6 \mathrm{~m} / \mathrm{min}(l e f t)$ and $7 \mathrm{~m} / \mathrm{min}$ (right)
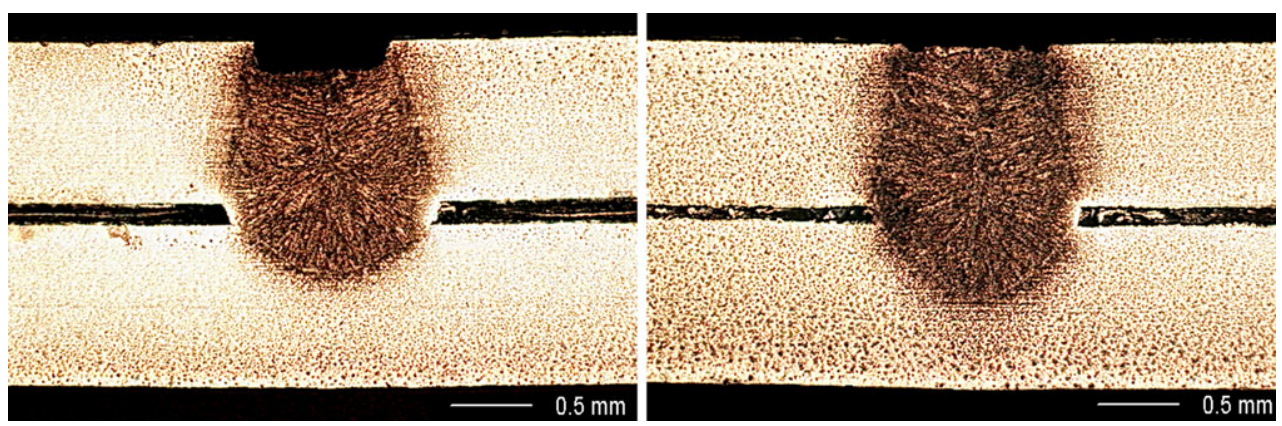

pected from Fig. 11, the penetration depth decreases slightly with decreasing feeding rate. Without closed-loop control, penetration depth would have increased close to the bottom of the work piece. Additional examples of compensated process drifts were published in [21].

\section{Conclusions}

In this paper the FPH image feature in zinc-coated steel sheets is regarded as a stochastic process. It is shown that the probability of its observation changes significantly when the penetration depth of the welding process reaches two interfaces: the bottom of the work piece in overlap or butt joints ("full penetration") and near the gap in lap joints ("partial penetration"). In both regimes, the probability varies almost linearly with the laser power. On the other hand, the transient response of the FPH to process variations is nonlinear and it varies with time. Despite these difficult properties, the relative frequency of FPH events can be used to control penetration depth. A special feedback strategy was developed to exploit the properties of the stochastic process for a closedloop system which guides the process near the minimum line energy power required for a certain penetration depth.

In the full penetration mode, this strategy guides the process near the minimum laser power necessary for a preset degree of penetration. This concept avoids excess laser power of typical $10 \%$ required in uncontrolled welding processes to compensate process drifts like polluted protection glasses, focal position, variations in speed or sheet thickness. The closed-loop system adapts the laser power automatically when process parameters change. As shown in previous publications, this concept holds for a large variety of different materials ranging from zinc-coated steel sheets with different thickness $[13,21]$ to aluminum of type AA 6014 [12].

In the partial penetration mode, the behavior of the FPH is even more complex. As the open-loop results show, the probability of the FPH shows hysteresis effects and it therefore depends on the path followed to reach a certain laser power. The behavior is also different at very low feeding rates. Whereas zinc-coated steel sheets show a very high peak, this peak can be much lower in other materials like aluminum [12]. Nevertheless, in zinc-coated steel sheets it is nearly independent from the gap size and the relative frequency of FPH events is an indirect measure for penetration depth. Thus, control in this regime enables for the first time partial penetration welding with controlled penetration depth-which is of particular interest in car body manufacturing. There, partial penetration welds allow welding in visible areas and this technique avoids the evaporation of zinc which reduces corrosion.

Acknowledgements This work was funded by the Baden-Württemberg Stiftung gGmbH within the project "Analoge Bildverarbeitung mit cellularen neuronalen Netzen (CNN) zur Regelung laserbasierter Schweißprozesse (ACES)".

Open Access This article is distributed under the terms of the Creative Commons Attribution License which permits any use, distribution, and reproduction in any medium, provided the original author(s) and the source are credited.

\section{References}

1. P. Berger, Gas Flow and Chemical Lasers, vol. 1810 (SPIE Press, Bellingham, 1992)

2. R. Fabbro, J. Phys. D, Appl. Phys. 43 (2010)

3. A. Otto, H. Koch, K.-H. Leitz, M. Schmidt, Phys. Procedia 12 (2011). doi:10.1016/j.phpro.2011.03.003

4. J. Müller-Borhanian, Kamerabasierte In-Prozessüberwachung beim Laserstrahlschweißen (Utz, München, 2009)

5. S. Kaierle, P. Abels, G. Kapper, C. Kratzsch, J. Michel, W. Schulz, R. Poprawe, in Proc. 20th International Congress on Applications of Lasers \& Electro-Optics ICALEO (2001)

6. F. Bardin, A. Cobo, J.M. Lopez-Higuera, O. Collin, P. Aubry, Appl. Opt. 44, 13-21 (2005)

7. J. Müller-Borhanian, Integration optischer Messmethoden zur Prozesskontrolle beim Laserstrahlschweissen (INESS): Abschlussbericht zum Verbundprojekt (Utz, München, 2005)

8. T. Klein, M. Vicanek, G. Simon, J. Phys. D, Appl. Phys. 29, 322332 (1996)

9. A. Blug, F. Abt, L. Nicolosi, F. Dausinger, H. Höfler, R. Tetzlaff, R. Weber, in Proc. 28th International Congress on Applications of Lasers \& Electro-Optics ICALEO (2009)

10. P. Strohm, A. Blug, D. Carl, H. Höfler, Tech. Messen 79, 3 (2012)

11. A. Rodríguez-Vázquez, R. Domínguez-Castro, F. JiménezGarrido, S. Morillas, A. García, C. Utrera, M.D. Pardo, J. Listan, R. Romay, in Cellular Nanoscale Sensory Wave Computing, ed. by C. Baatar, W. Porod, T. Roska (Springer, Boston, 2010) 
12. A. Blug, D. Carl, H. Höfler, F. Abt, A. Heider, R. Weber, L. Nicolosi, R. Tetzlaff, Phys. Procedia 12 (2011). doi:10.1016/j.phpro.2011.03.090

13. L. Nicolosi, A. Blug, F. Abt, R. Tetzlaff, H. Höfler, D. Carl, in Focal-Plane Sensor-Processor Chips, ed. by Á. Zarándy (Springer, New York, 2011)

14. A. Papoulis, S.U. Pillai, Probability, Random Variables, and Stochastic Processes (McGraw-Hill, Boston, 2009)

15. H. Hügel, T. Graf, Laser in der Fertigung: Strahlquellen, Systeme, Fertigungsverfahren, (Vieweg+Teubner Verlag GWV Fachverlage, Wiesbaden, 2009)

16. T. Forsman, J. Powell, C. Magnusson, J. Laser Appl. 13, 193-198 (2001)

17. R.G. Palmer, Adv. Phys. 31, 6 (1982)
18. A. Bollig, D. Abel, C. Kratzsch, S. Kaierle, in Proc. European Control Conference ECC (2003)

19. X. Na, Y.M. Zhang, Y. Liu, B. Walcott, IEEE Trans. Control Syst. Technol. 18 (2010). doi:10.1109/TCST.2009.2026163

20. A. Blug, F. Abt, L. Nicolosi, Patent Applikation: Verfahren zum Betrieb einer Vorrichtung und Vorrichtung. Applicant: BadenWürttemberg Stiftung. Patent Numbers DE 1020100139 14, WO 2011120672 A2, 2010

21. F. Abt, A. Blug, L. Nicolosi, F. Dausinger, H. Höfler, R. Tetzlaff, R. Weber, J. Laser Micro Nanoeng. 6, 2 (2011)

22. F. Abt, A. Heider, R. Weber, T. Graf, A. Blug, D. Carl, H. Höfler, L. Nicolosi, R. Tetzlaff, Phys. Procedia 12 (2011). doi:10.1016/j.phpro.2011.03.091 